\title{
NÍVEL DE ATIVIDADE FÍSICA E PREVALÊNCIA DE DOENÇAS CRÔNICAS DEGENERATIVAS EM FREQUENTADORES DE ÁREAS PÚBLICAS DA CIDADE DE INDIANA - SP.
}

Gabriel Teixeira De Brito, Guilherme Akio Tamura Ozaki, Thiago Alves Garcia, Lucas Da Silva Santos, Adriana Junqueira, Thainá Oliveira Felipe, Everton Alex Carvalho Zanuto, Fábio Rodrigo Gouveia Teixeira, Claudia De Matos Gomcalves Silva, Robson Chacon Castoldi, Regina Celi Trindade Camargo, José Carlos Silva Camargo Filho

Universidade do Oeste Paulista - UNOESTE, Faculdade de Educação Física, Presidente Prudente, SP. E-mail: gabs britoo@hotmail.com.

\section{RESUMO}

É possível observar o aumento da prática de atividades físicas em praças e áreas públicas das cidades brasileiras. Sabe-se que o exercício físico é um componente para estimular hábitos de vida saudável e contribuir na prevenção e manutenção da saúde. O objetivo do presente estudo foi investigar o nível de atividade física (NAF) e a prevalência de doenças crônicas em frequentadores de áreas públicas. Foram observados 70 frequentadores residentes da cidade de Indiana-SP. Os dados foram obtidos por meio "International Physical Active Questionnaire" (IPAQ - versão curta) que avalia o nível de atividade física (NAF) e a prevalência de doenças crônico-degenerativas (DCD) por um questionário baseado no "Standard Health Questionnaire". A prevalência de DCD e o NAF foram descritos por meio de analises da frequência de respostas. Foi verificado que $90 \%$ da população avaliada foi classificada como fisicamente ativa. Além disso, aproximadamente $40 \%$ da população está acima do peso e demonstraram $60 \%$ de prevalência de hipertensão arterial, $60 \%$ de doenças metabólicas endócrinas, $18 \%$ de doenças cardiovasculares e $27 \%$ de doenças osteomusculares. Dessa forma, é possível concluir que apesar de se apresentarem como fisicamente ativas, a presente amostra possui alta prevalência de doenças.

Palavras chave: Doenças Crônicas; Exercício Físico; Saúde; Prevenção de Doenças.

\section{PHYSICAL ACTIVITY LEVELS AND PREVALENCE OF CHRONIC DISEASE IN PUBLIC AREAS USERS IN INDIANA CITY - SP.}

\begin{abstract}
Is possible to observe that there has been an increase in the practice of physical activities in public areas. The physical exercise is a great component for healthy living and help in the prevention and maintenance of chronic-degenerative diseases. The objective of the present study was to observe the level of physical activity (PA) and the occurrence of chronic-degenerative diseases (DCD) in frequents public areas. There were 70 resident residents of the city of Indiana-SP. The data were collected through a questionnaire containing PA and occurrence of DCD. The PA and prevalence of chronic diseases was described through analyzes of frequency of responses. Was observed that $40 \%$ the population is higher body mass. Also, show $60 \%$ the Hypertension Prevalence, $60 \%$ metabolic endocrine disease, $18 \%$ cardiovascular disease and $27 \%$ musculoskeletal disease. Therefore, it is possible concluded that although be physically active the present people showed higher prevalence of DCD.
\end{abstract}

Keywords: Chronic Diseases; Exercise Activity; Health; Disease Prevention. 


\section{INTRODUÇÃO}

A prática de atividade física está crescendo nas cidades brasileiras, seja ela em ambientes fechados ou abertos, como é o caso das áreas públicas de lazer. Esta prática pode ser composta por exercícios que envolvam movimentos corporais com aplicação de uma ou mais aptidões físicas, além de contribuir na vida social e mental, de modo que trará como resultados na promoção de saúde ${ }^{1}$.

Os principais benefícios à saúde, advindos da prática de atividade física, referem-se aos aspectos antropométricos, neuromusculares, metabólicos e psicológicos. Nesse contexto, o Ministério da Saúde do Brasil criou o programa 'Academia da Saúde', para incentivo da atividade física. A expectativa de vida e desencadeamento de doenças crônicas, como Hipertensão Arterial, doenças Metabólico-Endócrinas e Osteomusculares, se dá em função do baixo nível de pratica de atividade física. Nesse caso, a prescrição de atividade física enquanto fator de prevenção de doença e melhoria da qualidade de vida ${ }^{2}$.

A interação da população, nas mais variadas dimensões que a atividade física oferece, possibilita a promoção de saúde e qualidade de vida. O aumento em $15 \%$ da produção diária de calorias, por cerca de 30 minutos de atividades físicas moderadas, pode fazer com que indivíduos sedentários passem a fazer parte do grupo de pessoas consideradas ativas, diminuindo, assim, suas chances de desenvolverem moléstias associadas à vida pouco ativa ${ }^{3}$.

Por sua vez, afirmam que a prática de exercícios físicos habituais, além de promover a saúde, influencia na reabilitação de determinadas patologias associadas ao aumento dos índices de morbidade e da mortalidade. A prática da atividade física influencia e é influenciada pelos índices de aptidão física, as quais determinam e são determinados pelo estado de saúde ${ }^{4}$.

Sendo assim, o objetivo do presente estudo foi analisar o Nível de Atividade Física (NAF) e a prevalência de Doenças Crônicas Degenerativas (DCD) nos frequentadores de áreas públicas da cidade Indiana- SP.

\section{MÉTODO}

\section{Amostra}

O estudo foi aplicado em frequentadores de áreas públicas "praça matriz" da cidade de Indiana - SP. Segundo dados do IBGE (2016), a cidade de escolhida para o estudo possui 4.936 habitantes. Nesse caso, após a realização do cálculo amostral, foram observados 70 indivíduos adultos, de ambos os sexos e de diferentes faixas etárias. A amostra foi obtida de forma aleatória, com coletas realizadas nos três períodos do dia (manhã, tarde e noite) com duração de duas semanas, em diferentes dias da semana, não sendo realizada nos finais de semana e períodos de férias ou datas comemorativas.

Instrumentos de medida

Foram utilizados os questionários de acordo com os aspectos a serem observados no presente estudo. Dessa maneira, foram constituídos de um questionário que avalia o Nível de Atividade Física (NAF) "International Physical Active Questionnaire" (IPAQ - versão curta) e a presença/ausência de doenças crônico degenerativa (DCD), baseado no Standard Health Questionnaire, dividido em quatro principais grupos de doenças (Hipertensão Arterial, Doenças Metabólicas-Endócrinas, Cardiovasculares e Osteomusculares).

A anamnese individual obteve informações do praticante de modo geral. No mesmo, teve perguntas como idade, sexo, peso corporal, estatura, nível de escolaridade e hábitos cotidianos.

Estado nutricional

A classificação do estado nutricional foi obtida pelo cálculo da razão da Massa Corporal pelo quadrado da Estatura (Peso Corporal/Estatura ${ }^{2}$ ). Os indivíduos foram classificados a partir das recomendações da Organização Mundial de Saúde.

Ética em pesquisa em seres humanos 
Para a realização da coleta de dados, o projeto do presente estudo foi encaminhado e aprovado, antecipadamente à coleta, ao comitê de ética em pesquisa em seres humanos da Universidade do Oeste Paulista - UNOESTE.

Análise Estatística

Após a obtenção dos dados, foi utilizada a análise de frequência para a caracterização da amostra. Neste caso, foram descritas as variáveis obtidas pelos questionários utilizados no presente estudo. Tal procedimento foi realizado com o Software SPSS $22.0{ }^{\circledR}$.

\section{RESULTADOS}

Quando analisado o índice de massa corporal (IMC), foi possível verificar que pouco mais da metade da população avaliada apresenta eutrofia (Tabela 1).

Tabela 1. Índice de Massa Corporal.

\begin{tabular}{ccc}
\hline & Frequência & Porcentagem \\
\hline Eutrofia & 38 & $54,3 \%$ \\
Sobrepeso & 20 & $28,6 \%$ \\
Obesidade & 12 & $17,1 \%$ \\
& & \\
Total & 70 & 100,0 \\
\hline
\end{tabular}

Já quando analisada a escolaridade, é possível notar que a maioria dos participantes possuem ensino médio e curso superior, respectivamente (Tabela 2).

Tabela 2. Escolaridade

\begin{tabular}{ccc}
\hline & Frequência & Porcentagem \\
\hline Fundamental & 1 & $1,4 \%$ \\
Básico & 8 & $11,4 \%$ \\
Médio & 30 & $42,9 \%$ \\
Superior & 26 & $37,1 \%$ \\
Pós Graduados & 5 & $7,1 \%$ \\
Total & 70 & $100 \%$ \\
\hline
\end{tabular}

Foi verificado também que a maioria dos entrevistados foram classificados como fisicamente ativos (tabela 3).

Tabela 3. Nível de Atividade Física

\begin{tabular}{ccc}
\hline & Frequência & Porcentagem \\
\hline Insuficientemente Ativo & 7 & $10 \%$ \\
Fisicamente Ativo & 63 & $90 \%$ \\
Total & 70 & $100 \%$ \\
\hline
\end{tabular}

Já quando observada a prevalência de doenças, nota-se que a maioria da população observada mostra a ocorrência de hipertensão arterial (Tabela 4). 
Tabela 4. Prevalência de Hipertensão Arterial

\begin{tabular}{ccc}
\hline & Frequência & Porcentagem \\
\hline Ausência Hipertensão Arterial & 28 & $40 \%$ \\
Presença Hipertensão Arterial & 42 & $60 \%$ \\
Total & 70 & $100 \%$ \\
\hline
\end{tabular}

Constatou-se que a minoria da amostra se apresenta livre de Doenças Metabólicas Endócrinas (DME) (tabela 5). Nesse caso, 60\% dos entrevistados relataram presença de uma ou mais patologias.

Tabela 5. Prevalência de Doenças Metabólicas Endócrinas (DME)

\begin{tabular}{ccc}
\hline Presença/Ausência & Frequência & Porcentagem \\
\hline Ausência DME & 28 & $40 \%$ \\
Presença de 1 DME & 17 & $24,3 \%$ \\
Presença de 2 DME & 21 & $30 \%$ \\
Presença de 3 DME & 3 & $4,3 \%$ \\
Presença de 4 DME & 1 & $1,4 \%$ \\
Total & 70 & $100 \%$ \\
\hline
\end{tabular}

Foi verificado também que a maioria da população estudada não apresenta Doenças Cardiovasculares (Tabela 6). No entanto, $18,5 \%$ da população demonstrou ocorrência de uma ou mais doenças.

Tabela 6. Prevalência de Doenças Cardiovasculares

\begin{tabular}{ccc}
\hline & Frequência & Porcentagem \\
\hline $\begin{array}{c}\text { Ausência de Doenças } \\
\text { Apresenta Doença }\end{array}$ & 57 & $81,5 \%$ \\
$\begin{array}{c}\text { Cardiovascular } \\
\text { Mais de uma Doença } \\
\text { Cardiovascular } \\
\text { Total }\end{array}$ & 5 & $7,1 \%$ \\
\end{tabular}

Quando analisada a ocorrência de Doenças Osteomusculares, foi verificado que a maioria dos entrevistados apresentavam ausência para este grupo de doenças (Tabela 7).

Tabela 7. Prevalência de Doenças Osteomusculares

\begin{tabular}{ccc}
\hline & Frequência & Porcentagem \\
\hline Ausência de Doenças & 51 & $72,9 \%$ \\
Presença de uma Doença & 9 & $12,9 \%$ \\
$\begin{array}{c}\text { Presença de duas } \\
\text { Doenças }\end{array}$ & 8 & $11,4 \%$ \\
$\begin{array}{c}\text { Presença de ter ou mais } \\
\text { Doenças } \\
\quad \text { Total }\end{array}$ & 2 & $2,9 \%$ \\
& 70 & $100 \%$
\end{tabular}

Quando verificada a amostra a partir do sexo, foi verificada ligeira maioria de indivíduos do sexo feminino. 
Tabela 8. Distribuição por Sexo

\begin{tabular}{ccc}
\hline & Frequência & Porcentagem \\
\hline Masculino & 30 & $42,9 \%$ \\
Feminino & 40 & $57,1 \%$ \\
Total & 70 & $100 \%$ \\
\hline
\end{tabular}

Por último, foi verificada a distribuição de participantes a partir da idade. Neste caso, percebeu-se que a maioria se enquadra como adultos jovens, ou seja, com idade inferior a 30 anos.

Tabela 9. Distribuição amostral por diferentes faixas etárias.

\begin{tabular}{ccc}
\hline & Frequência & Porcentagem \\
\hline Menor de 30 anos & 40 & $57,1 \%$ \\
Entre 30 a 50 anos & 14 & $20 \%$ \\
Maior de 50 anos & 16 & $22,9 \%$ \\
Total & 70 & $100 \%$ \\
\hline
\end{tabular}

\section{DISCUSSÃO}

Após a obtenção dos dados, foi verificado que mais de $40 \%$ da amostra analisada apresenta excesso de peso. Estudos mostram que a condição de sobrepeso e obesidade está diretamente relacionada ao surgimento de doenças ${ }^{5}$. Tal fato pode ser um indicativo preocupante para amostra analisada.

De acordo com averiguação dos dados, pode-se observar que maioria dos praticantes completaram os ciclos educacionais, sendo $42 \%$ concluíram o ensino médio e $37 \%$ ensino superior. Isso expõe que a escolaridade pode ter influência na pratica de atividade física e cuidado com a saúde.

O sedentarismo é visto como fator de risco primário para as doenças cardiovasculares. Nesse caso, torna-se fundamental a identificação dos determinantes da atividade física, para em seguida, serem propostos modelos teóricos para incentivar a adoção e manutenção da prática de atividades físicas, bem como, estratégias para incentivar a população a adotar o estilo de vida ativo fisicamente ${ }^{6,9}$.

Se tratando de doenças crônico-degenerativas. Foi verificado que a prevalência na população analisada foi de $60 \%$ para Hipertensão Arterial, 60\% para doenças Metabólicas Endócrinas, aproximadamente $20 \%$ para doenças Cardiovasculares e $27 \%$ para doenças Osteomusculares. Tal achado demonstra números elevados de doenças e retrata o risco de saúde à população observada. Além disso, estudos demonstram que essa ocorrência poderia ser minimizada ou evitada por meio da realização de atividades físicas ${ }^{7-9}$.

Foi observado ainda que maior número de adeptos do sexo feminino (57,1\%). Além disso, entre todos os entrevistados, adultos e jovens entre 18 e 30 anos, foi sobressalente entre os demais, chegando a $57,1 \%$. Nesse contexto, a prática de atividade física pode ser considerada como meio preventivo no surgimento de doenças, principalmente durante o processo de envelhecimento, período no qual a população se torna mais susceptível ao desenvolvimento de doenças crônicas ${ }^{10-12}$.

Por fim, o presente estudo contribui com a literatura ao identificar o perfil dos praticantes de atividade física em frequentadores de áreas públicas. No entanto, limitações devem ser consideradas, como a área de estudo (área pública) e métodos de mensuração utilizados. Estudos futuros que investiguem diferentes áreas ou participantes, podem vir a contribuir com os achados expostos até o momento. 


\section{CONCLUSÃO}

Conclui-se que aproximadamente $90 \%$ da população analisada pode ser considerada como "fisicamente ativa". No entanto, $40 \%$ dos avaliados estão acima do peso. Além disso, demonstraram $60 \%$ de prevalência de hipertensão arterial, $60 \%$ de doenças metabólicas endócrinas, $18 \%$ de doenças cardiovasculares e $27 \%$ de doenças osteomusculares.

\section{REFERÊNCIAS}

1.Guedes DP, Guedes JERP. Exercício físico na promoção da saúde. Londrina: Midiograf 1995.

2.Matsudo SM, Matsudo VKR. Evidências da importância da atividade física nas doenças cardiovasculares e na saúde. Rev Diag Trat 5(2):10-17, 2000.

3.Matsudo VKR. Vida ativa para o novo milênio. Rev Oxid, 1999.

4. Carvalho T, Guedes DP, Silva JG. Orientações Básicas sobre Atividade Física e Saúde para Profissionais das Áreas de Educação e Saúde. Brasília: Ministério da Saúde e Ministério da Educação e do Desporto 1996.

5. Pitanga FJG. Epidemiologia, atividade física e saúde. Rev Bras.Ciên Mov 10 (3): 49-54, 2002.

6. Matsudo SM. Atividade física na promoção da saúde e qualidade de vida no envelhecimento. Rev bras Educ Fís Esp 20(5):135-137, 2006.

7.Neto TLB. Atividade Física e Qualidade de vida. In: Anais do I Congresso Centro-Oeste de Educação Física, Esporte e Lazer; Brasília, setembro de 1999.

8. Matsudo SM, Matsudo VKR, Barros Neto TL. Efeitos benéficos da atividade física na aptidão física e saúde mental durante o processo de envelhecimento. Rev Bras Ativ Fis Saúde 5(2), 2000.

9.Revista do Sistema Único de Saúde do Brasil. Rev Epidemiol Serv Saúde 22(1), 2013.

10. Serrano J, Faustino A, Rato V, Petrica J, Paulo R, Mendes P, et al. "O perfil comum dos programas de atividade física das academias séniores. Rev Cienc del Deporte 11.5: 135-136, 2015.

11.Fernandes EC. Qualidade de vida no trabalho: como medir para melhorar. Salvador: Casa da Qualidade; 1996.

12. Matsudo V, Guedes J, Matsudo S, Andrade D, Araujo T, Oliveira L et al. The mobile management of the ecological model to promote physical activity. Perspectives 6: 427-44, 2004. 